\title{
$=-$ From the World Health Organisation \\ Assessing the health effects of tobacco use in developing countries and eastern Europe
}

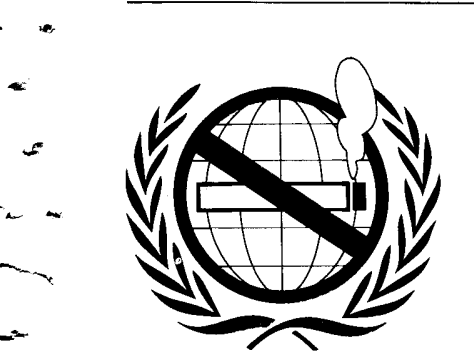

In all fields of public health and disease control, quantifiable estimates of the mortality and morbidity due to epidemics are invaluable for determining health policies and strategies, and for monitoring the effectiveness of control measures. The need for reliable, timely and sensitive estimates, and the role of epidemiology in providing them, has been appreciated since the era of infectious diseases and is no less relevant today when tobacco, rather than cholera, tuberculosis or injuries, is the leading cause of adult death in the industrialised world.

While estimates of the current effects of past tobacco use are helpful to place the epidemic in perspective, it is projections of the future effects of current smoking patterns that are likely to be of much greater relevance for health policies. Many of the future premature deaths among those who smoke today, or those who might do so shortly, can be prevented by policies which discourage smoking in youth and/or contribute to a reduction in risk through smoking cessation. The World Health Organisation (WHO), in collaboration with epidemiologists at Oxford University, United Kingdom, have estimated that there are already about three million annual deaths from tobacco in the world, of which about one-third are in less developed countries. However, the WHO collaborative group have projected that, on current smoking trends, annual worldwide mortality from smoking might eventually reach ten million per year, two-thirds of whom might be in developing countries!

While the relative order of magnitude of tobacco-attributable mortality in developed countries today (about $20 \%$ of all deaths) has been established with some confidence by the WHO group, the prediction of a rapid increase in tobacco-attributable deaths in less developed countries needs to be monitored reliably. This prediction is based not on direct evidence from such countries (evidence which, by and large, does not yet exist), but on indirect extrapolation from studies carried out in the very different epidemiological environments of developed countries.

The most reliable way of monitoring both the current level and the evolution of the epidemic of death from tobacco is through prospective studies of $100000-200000$ or more adults. Smoking behaviour and related health characteristics are assessed in a baseline questionnaire and the subsequent mortality of smokers and non-smokers is then monitored for several years as the epidemic matures. Several such studies have been carried out in developed countries, the largest being the two American Cancer Society studies in the 1960s and 1980s, each involving over one million adults (Cancer Prevention Study I and II). The importance of extended follow-up is well demonstrated by the British Doctors Study in which survival has been monitored since 1951, during which time the relative risks of death for smokers compared with non-smokers have increased progressively. Prospective studies facilitate the assessment of smoking-attributable risks from several causes of death and measure the impact of co-factors such as alcohol, blood pressure, body mass index, occupation, etc, which may interact synergistically with tobacco to increase disease risks, presumably differentially in different populations.

Recognising the need to monitor more reliably the health effects of the massive increase in tobacco use in developing countries, particularly among men, the WHO, in collaboration with Oxford University, has established a network of prospective studies in developing countries. Overall study design, pilot studies, common analytical strategies, and technical assistance to countries are coordinated, and it is expected that the results will increasingly stimulate action to control the epidemic by providing more reliable, locally relevant and comprehensive information on the health effects of tobacco.

Eventually, it is expected that prospective studies which will monitor the evolution of the epidemic into the twenty-first century will be established in all developing regions of the world, provided funds are available. At present, a number of studies are being implemented within the auspices of the network, as summarised below:

China: 500000 adults have been surveyed in Beijing and 80 other areas of the country. Follow-up is being monitored through the Disease Surveillance Points system of the Chinese Academy of Preventive Medicine. In addition, a second cohort of 200000 adults in Shanghai has been followed up since 1982 .

Cuba: Using the records of the extensive Cuban health care network, the relative survival of 100000 male smokers and non-smokers will be followed up. A pilot study is nearing completion and, depending on the evaluation of results, the main study is expected to commence before the end of 1993 .

India: Over 55000 adults in Bombay have been recruited so far, with a target of 100000 or more. Recruitment is proceeding rapidly and follow-up will be on the basis of civil registration records in Bombay. This study is being carried out in collaboration with the International Agency for Research on Cancer in Lyon, France. 
Mexico: A study of over 100000 households (including blood samples) is being carried out in Mexico City with follow-up through vital registration. The results of this study will shed further light on the interaction of smoking with blood cholesterol levels in a developing country setting.

Poland: A study of 200000 adults (including blood samples) is being undertaken. Pilot studies in several regions of the country have been carried out and it is expected that the main study will commence shortly. Poland is an extremely important surveillance site for monitoring tobacco-attributable deaths because male lung cancer death rates are already at levels comparable to the UK and the US at the height of their epidemics, blood cholesterol levels are high, and well over half of all Polish men smoke.

In addition to these sites, small exploratory pilot studies are being carried out, or are planned, for Trivandrum and Vellore (India), Buenos Aires (Argentina), and Ismailia (Egypt). Preliminary discussions have been held with scientists in Brazil, Indonesia, and Thailand in the hope of establishing similar studies in these countries, countries where more than half of all adult males are regular smokers.

By drawing on expertise from countries where prospective studies have been carried out and used to inform health policies, WHO is uniquely placed to coordinate the technical support for these studies and to seek financial support for them. Their significance and urgency for international public health lie in the alarming predictions about tobaccoattributable deaths in developing countries, predictions which will be much more reliably informed in the future as the results of these studies become available.

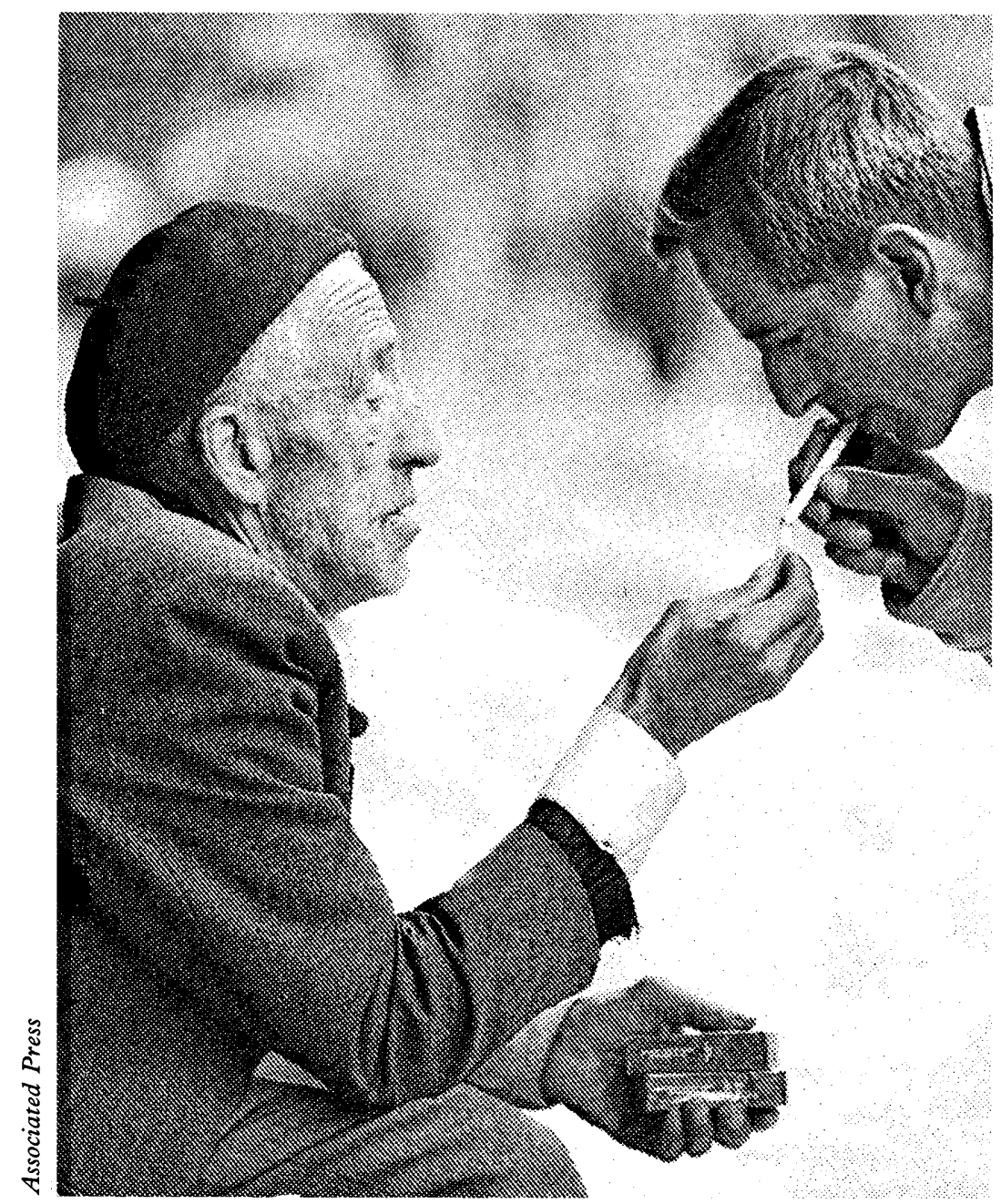

In Sarajevo, Bosnia and Herzegovina, people complain more about the scarcity of cigarettes than about the scarcity of food, water, or even ammunition. Cigarettes can cost from $\$ 5$ to $\$ 10$ a pack. A black market trader (shown above on the left), lights a cigarette for a passer-by. One smuggler reported "You can buy a kilo of wheat flour, enough to feed yourself for 10 days, for the price of just one pack of cigarettes". According to a 30-year-old Bosnian Army soldier, "Smokers will tell you they can survive on only one meal a day if they can smoke". (Source: New York Times,
5 September 1993.) 